\title{
Excitation of voltage oscillations in an induction voltage adder
}

\author{
Nichelle Bruner, Thomas Genoni, Elizabeth Madrid, and Dale Welch \\ Voss Scientific, LLC, Albuquerque, New Mexico 87108, USA \\ Kelly Hahn and Bryan Oliver \\ Sandia National Laboratories, Albuquerque, New Mexico 81185, USA
}

(Received 8 April 2009; published 1 July 2009)

\begin{abstract}
The induction voltage adder is an accelerator architecture used in recent designs of pulsed-power driven x-ray radiographic systems such as Sandia National Laboratories' Radiographic Integrated Test Stand (RITS), the Atomic Weapons Establishment's planned Hydrus Facility, and the Naval Research Laboratory's Mercury. Each of these designs relies on magnetic insulation to prevent electron loss across the anode-cathode gap in the vicinity of the adder as well as in the coaxial transmission line. Particle-incell simulations of the RITS adder and transmission line show that, as magnetic insulation is being established during a pulse, some electron loss occurs across the gap. Sufficient delay in the cavity pulse timings provides an opportunity for high-momentum electrons to deeply penetrate the cavities of the adder cells where they can excite radio-frequency resonances. These oscillations may be amplified in subsequent gaps, resulting in oscillations in the output power. The specific modes supported by the RITS- 6 accelerator and details of the mechanism by which they are excited are presented in this paper.
\end{abstract}

DOI: 10.1103/PhysRevSTAB.12.070401

PACS numbers: 41.85.Ja, 52.65.Rr, 84.70.+p

\section{INTRODUCTION}

The induction voltage adder (IVA) is an architecture for connecting a number of voltage drivers in series in order to propagate their summed voltages down a central transmission line. Details of the IVA and induction accelerator family are presented in Ref. [1]. This architecture is used in recent designs of pulsed-power radiographic systems such as Sandia National Laboratories' Radiographic Integrated Test Stand (RITS) [2], the Atomic Weapons Establishment's Hydrodynamic Radiographic Facility (HRF) [3], and the Naval Research Laboratory's Mercury [4]. RITS-6, the most recent RITS configuration, delivers 7.8 MV with $186 \mathrm{kA}$ of current in $70 \mathrm{~ns}$ for its lowimpedance transmission line [5]. Mercury is designed to deliver $6 \mathrm{MV}$ and $360 \mathrm{kA}$ in $50 \mathrm{~ns}$, while HRF is designed for $14 \mathrm{MV}$ and $100 \mathrm{kA}$ in $60 \mathrm{~ns}$. In each of these designs, the adder output polarity is (or can be configured to be) negative such that the inner conductor of the adder section becomes the cathode of the coaxial transmission line. These high-powered systems contain high electric field stresses which result in electron emission along almost the entire length of the cathode, including in the IVA section, and rely on magnetic insulation to prevent free electrons from crossing the coaxial gap. This configuration is referred to as a magnetically insulated transmission line (MITL) [6,7]. The MITL terminates in an electron diode in which electrons striking a high- $Z$ anode produce $\mathrm{x}$ rays.

During the pulse rise as magnetic insulation is being established, some electron loss occurs across the coaxial gap. The magnitude of this loss in the adder section is a function of the local line voltage and geometry, but is not disruptive of the voltage pulse under nominal operating conditions. However, under nonideal operating conditions, large radio-frequency (rf) oscillations have been measured in the cavities of the RITS- 6 induction cells which are shown in this paper to be related to the free electron flow. Since the induction cells are coupled to the diode through the central transmission line, as shown in the RITS-6 schematic in Fig. 1, these oscillations can propagate down the MITL and manifest as oscillations in the output power. These oscillations occur early in the pulse, are independent of the load configuration, and are correlated with poorly timed induction cell pulses.

Figure 2 shows data from a representative RITS-6 shot demonstrating oscillations. In this shot, the transmission line terminates in a hollow cylinder; this is a nonradiographic load referred to as a blade diode. Figure 2(a) shows the temporal profiles of four current $B$-dot monitors located inside the cavities and Fig. 2(b) shows the corresponding measured $\mathrm{x}$-ray signal generated from the diode region. The $\mathrm{x}$-ray dose rate is recorded using a pin diode located approximately $3 \mathrm{~m}$ downstream from the electron beam converter material.

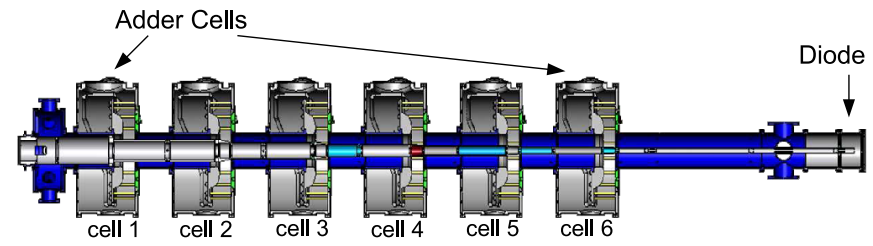

FIG. 1. (Color) Cross section of the RITS-6 induction cell cavities and the MITL. 

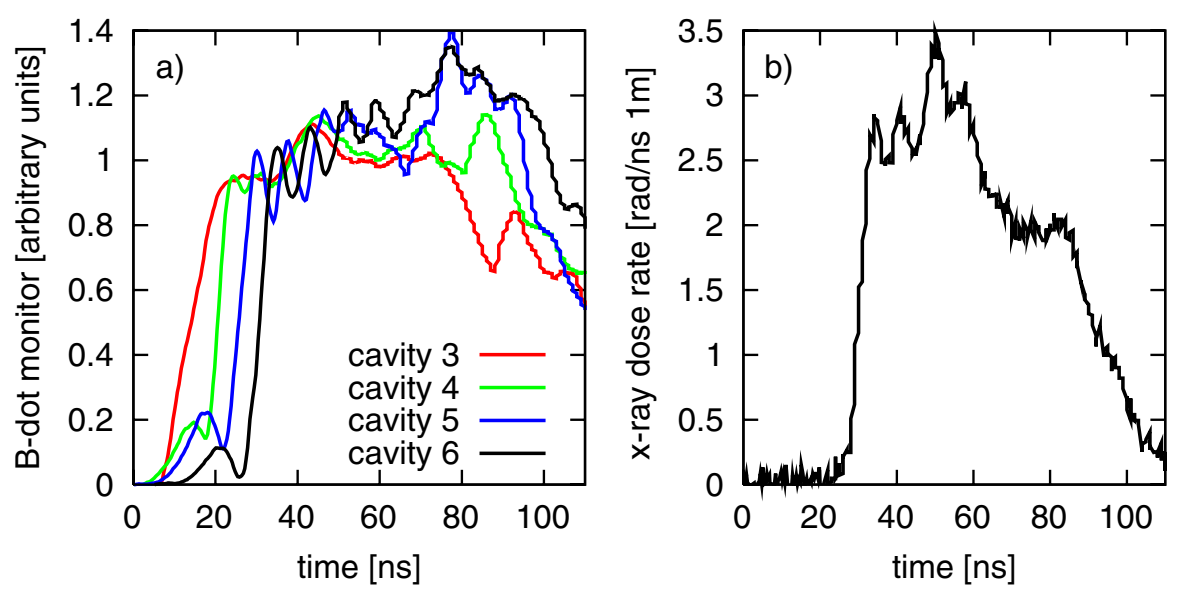

FIG. 2. (Color) Data demonstrating rf oscillations (120 MHz) on RITS-6 for a nonradiographic blade diode load (shot 13). B-dot probes located in induction cavities 3-6 are shown in (a). See the schematic in Fig. 1 for the relative locations of these cavities. The $B$-dot monitors shown are located 180 degrees from the radial feed. The x-ray dose rate recorded using a pin diode located approximately $3 \mathrm{~m}$ downstream from the electron beam converter material is shown in (b).

This effect is reproduced in detailed particle-in-cell simulations of RITS-6 using realistic accelerator pulse profiles and geometry. The simulations demonstrate that, if the relative timing of the pulse firings of some inductive cells is delayed, high-momentum electrons may deeply penetrate the cavities of the adder cells where they can excite a cavity rf mode. This resonance may couple to the beam and experience progressive amplification as it propagates past successive IVA cells. Thus, while this study focuses on RITS-6, other IVA-based accelerators would likely also be susceptible to this effect.

Details of the design of the RITS- 6 accelerator and the parameters used in modeling are presented in Sec. II. Calculations of the resonant modes for the RITS cavities are presented Sec. III, and the mechanism for mode excitation is discussed in more detail in Sec. IV.

\section{RITS-6 IVA DESIGN}

An IVA is composed of individual induction cells connected in series along a central cylindrical stalk which forms the inner conductor of a coaxial transmission line. Each cell transfers power from one or more individual voltage drive sources to the stalk. The pulses from each pulse-forming line are timed to arrive nearly simultaneously at the outlet of the adder section to form a single pulse at the load.

As mentioned in Sec. I, high-power IVA architectures such as RITS contain electric field stresses in the transmission line which exceed the threshold above which electrons are emitted [8], resulting in free electrons in the anode-cathode gap. In a MITL, sufficiently high line currents generate a magnetic field which constrains the trajectories of these electrons, preventing them from crossing the gap. Detailed descriptions of this magnetic cutoff and the relationships between the cathode boundary and sheath currents in equilibrium are found in Refs. $[9,10]$.

Figure 1 shows a cross section of RITS- 6 with the six induction cells and a tapered cathode stalk. The RITS IVA cell conducts an input drive pulse through a single port (radial feed) on its surface and into an oil-filled cavity. The pulse is then transferred to the adder bore in a vacuum region commonly referred to as the cell cavity. Each IVA cell is connected to the common transmission line by an azimuthal transmission line designed to distribute the pulse as symmetrically as possible around the center conductor. The level of pulse symmetry achieved in the transmission line has been previously studied [11]. Voltage isolation between each cell is established by use of a ferromagnetic core [1].

These IVA features are included in the simulations of the RITS-6, as shown in the simulation geometry in Fig. 3. The simulations are conducted in 3D $(x, y, z)$ using the fully relativistic particle-in-cell code LSP [12]. A symmetry plane is located at $x=0$, as shown in Fig. 3, to reduce computation time. (Oscillation results were verified with a full 3D simulation.) Particle emission is not included in the studies in Sec. III which determine the cavity resonant modes. In the studies of mode excitation in Sec. IV, space-charge-limited (SCL) electron emission is modeled along the entire length of the cathode with a field-stress threshold of $250 \mathrm{kV} / \mathrm{cm}$. An implicit time biasing algorithm [13] is used in the particle simulations to damp numerical high-frequency electromagnetic noise which may occur artificially from the large charge-to-mass ratios of the emitted electron macroparticles.

The depiction of the electron sheath surrounding the cathode in Fig. 3 is a snapshot of the flow after magnetic insulation has been established. Figure 4 shows electron flow during the pulse rise, while emission is still being initiated along the transmission line. This is normal elec- 


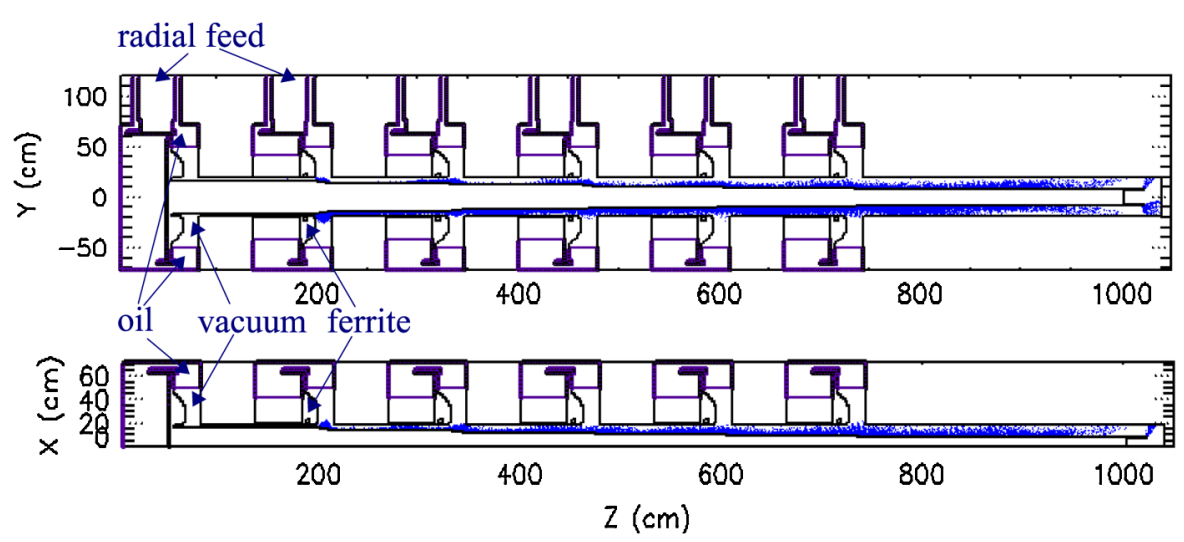

FIG. 3. (Color) Cross sectional views $(x, z$ and $y, z)$ of the 3D simulation geometry including the six induction cells. Electron macroparticles, shown in blue in the coaxial gap, illustrate flow after magnetic insulation. This geometry is derived from the RITS-6 schematic in Fig. 1.

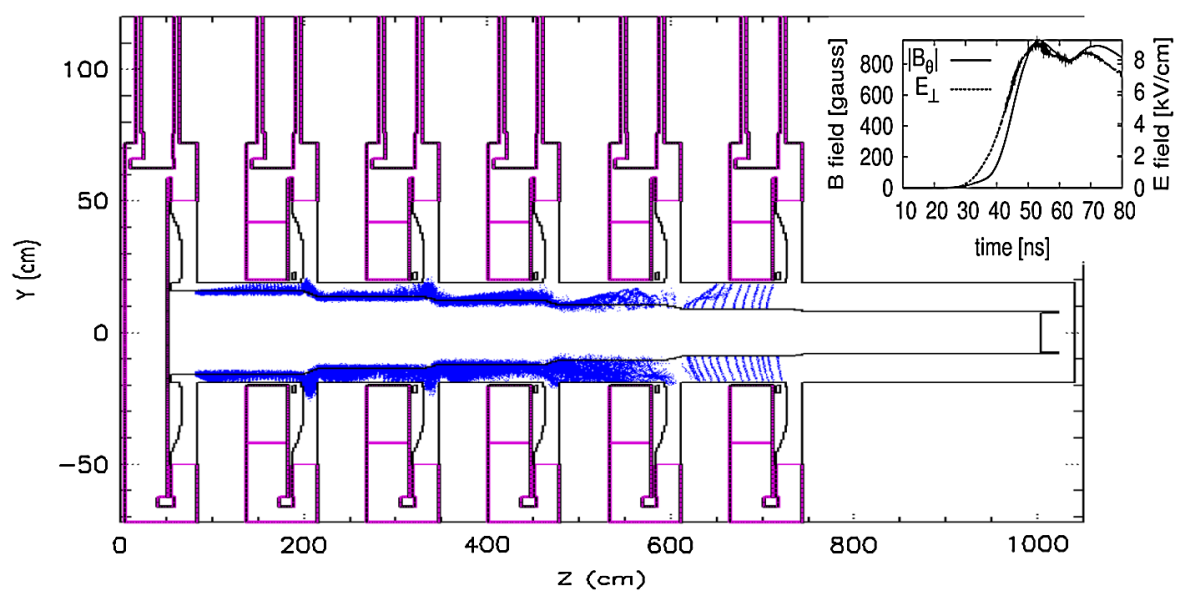

FIG. 4. (Color) Cross section $(y, z)$ of the $3 \mathrm{D}$ simulation geometry at $45 \mathrm{~ns}$ into the pulse for nominal pulse timings. Electron macroparticles (in blue) illustrate flow as magnetic insulation is being established. The field plot in the inset shows the time history of the $E$ and $B$ fields at $r=35 \mathrm{~cm}$ in cavity 4 , for reference.

tron flow for nominal cell timings. The electrons flow radially until the onset of insulation and some electrons are seen to penetrate the cavities of cells 2 and 3 by a few centimeters.

\section{IVA RESONANT MODES}

The frequency responses of the IVA vacuum cavities are calculated numerically since the complicated geometry of the cells makes an analytical calculation intractable. Instead, the resonance frequencies are determined by placing a dipole current source in the vacuum section of cavity 2. The 3-cm-long dipole source is oriented in the transverse direction and occupies the space from $z=206$ to $208 \mathrm{~cm}$ in Fig. 3.

Resonance frequencies are first determined crudely by gradually increasing the dipole frequency from 67 to $759 \mathrm{MHz}$ over $2.2 \mu \mathrm{s}$, while keeping the amplitude constant, to allow resonances to develop in the entire simula- tion space. The response of the transverse component of the electric field is recorded for each cavity at positions located 180 degrees in azimuth from the source. The spectra from cavities 4 through 6 are plotted in Fig. 5 . While these spectra appear quite crowded, strong azimuthally symmetric TEM resonances are seen in all three cavities near 120, 340, and $510 \mathrm{MHz}$. (The use of a symmetry plane in the simulation restricts the frequency response, removing significant asymmetric modes at 80 , 225 , and $250 \mathrm{MHz}$, as verified in a full 3D treatment.)

The mode qualities, or sharpness of response, of the resonances identified in Fig. 5 are determined in simulations using a dipole source with a much narrower and shorter frequency ramp. The dipole is turned off after the ramp and the decay of the fields is observed. For the mode near $120 \mathrm{MHz}$, the frequency ramp is 116 to $141 \mathrm{MHz}$ over $100 \mathrm{~ns}$. The dipole source is then turned off and the fields are recorded for $2 \mu \mathrm{s}$. A fast Fourier transform (FFT) is performed on the fields in each cavity over two time 


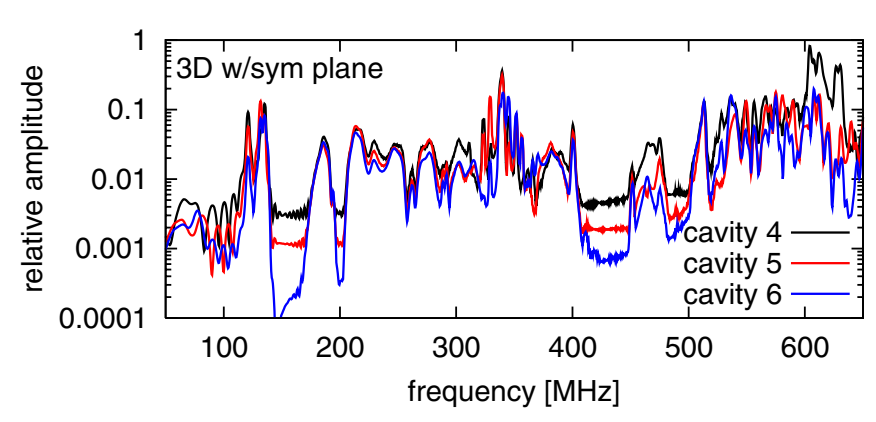

FIG. 5. (Color) Field resonances of the IVA cavities determined using the full RITS-6 geometry.

periods beginning after the dipole source has been turned off. The time periods, which are chosen to have sufficient separation from the dipole drive to observe decay, are $200 \mathrm{~ns}-2 \mu \mathrm{s}$ and $400 \mathrm{~ns}-2 \mu \mathrm{s}$. The dipole waveform is plotted in Fig. 6(a), showing the dipole drive terminating at 100 ns. Since the full simulation time is not shown, only the beginning of the FFT window is marked. The FFT results are shown in Fig. 7. The resonance near $120 \mathrm{MHz}$ is clearly very long lived in each cavity. The peaks in Fig. 7 are located at $123.8 \mathrm{MHz}$ for cells 1 and 2, 121.9 MHz for
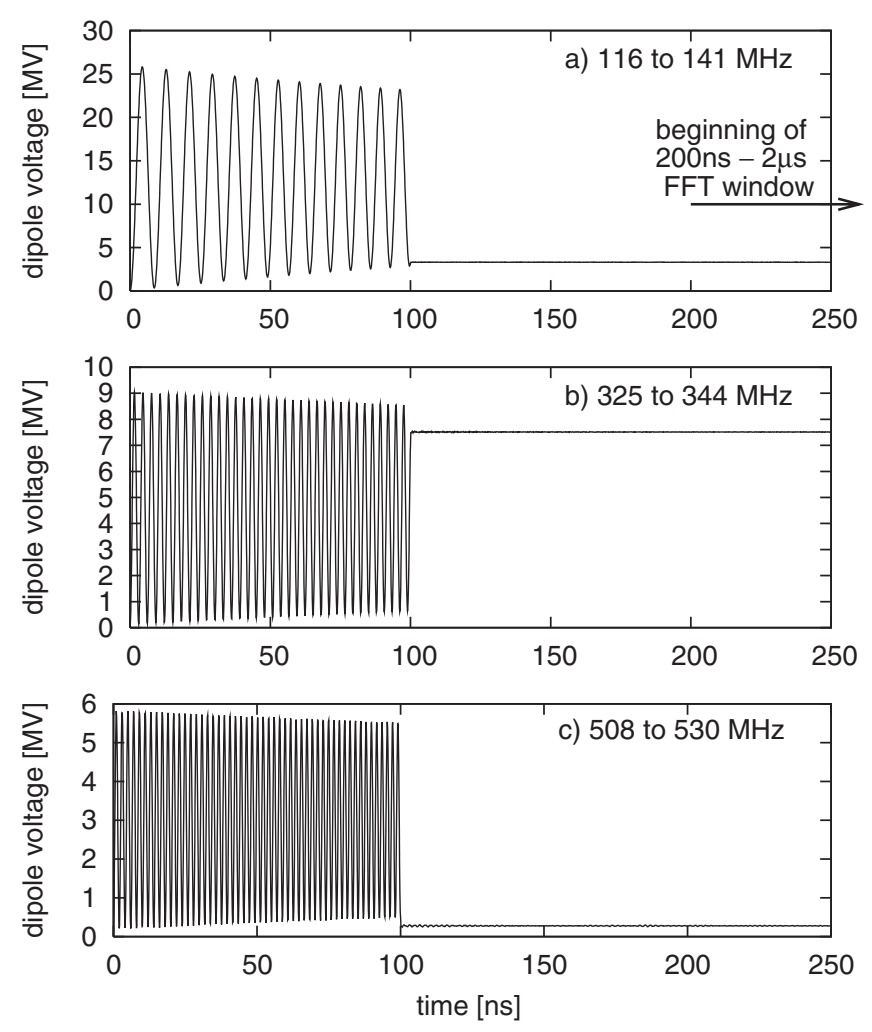

FIG. 6. Voltages of the applied dipoles used to calculate the resonance mode qualities. Plot (a) represents the frequency ramp from 116 to $141 \mathrm{MHz}$ over $100 \mathrm{~ns}$. Plots (b) and (c) are the frequency ramps from 325 to $344 \mathrm{MHz}$ and 508 to $530 \mathrm{MHz}$, respectively. The arrow marks the beginning of the period over which the resonance qualities are calculated. The plots do not cover the full $2 \mu \mathrm{s}$ of the simulation time. cell 3, and $120.7 \mathrm{MHz}$ for cells 4 through 6. For cells appearing to share a resonance frequency, the differences may be below $630 \mathrm{kHz}$, the frequency resolution used in the Fourier transform. The mode quality is defined as $Q=$ $\omega_{0} / \Gamma$ where $\Gamma$ is the full width at half maximum of the power spectrum. Calculating $\Gamma$ from the square of the $|E(\omega)|$ spectra for $t>200 \mathrm{~ns}$ in Fig. 7, the values of $Q$ range from 200 to 400 .

For the mode near $340 \mathrm{MHz}$, the frequency ramp is 325 to $344 \mathrm{MHz}$ over $100 \mathrm{~ns}$, shown in Fig. 6(b). The resonance occurs at $354 \mathrm{MHz}$ and is not as long lived as the $121 \mathrm{MHz}$ resonance, with a range of $Q$ values from 70 to 200. The third frequency ramp of 508 to $530 \mathrm{MHz}$ [Fig. 6(c)] produces broad, short-lived peaks near $523 \mathrm{MHz}$. The range of $Q$ values for these is 25 to 100 . Not surprisingly given these values of $Q$, both of these frequency ramps excited the lower modes.

\section{MODE EXCITATION}

The mechanism identified in simulation for exciting strong IVA cavity resonances is a transverse impulse to the $E$ field given when a strong transient radial current deeply penetrates an IVA cavity. Conditions for this occur when a sufficient delay is introduced between the pulse firings of the upstream IVA cells relative to the downstream cells. In this case, the field-stress threshold for SCL emission may be exceeded in the vicinity of a cell, or electron flow may arrive from upstream, so that free electrons have reached the cylindrical wall of the cell before it fires. The available high transverse-momentum electrons are able to reach large radii in the cavity as the cell fires. The mode is excited by an exchange of energy with these highmomentum electrons and may then progressively amplify as it propagates past successive IVA cavities.

The mechanism of mode excitation and its progressive amplification are demonstrated in a 3D simulation of RITS-6 operation. In this simulation, a pulsed voltage feed is applied to each IVA cell and SCL electron emission is enabled along the MITL cathode with a $250-\mathrm{kV} / \mathrm{cm}$ threshold. The voltage feed applied to each cell is an idealized pulse with a 12.0-ns rise to $1.4 \mathrm{MV}$ and a 55.0ns plateau. The nominal timing for cell firings in simulation is a 3.3-ns delay between successive cells. A delay of $7.7 \mathrm{~ns}$ between the firings of cells 3 and 4 allows free electrons to reach large radii in cavities 4 and 5. (The exact timings used in this simulation are 0, 0.3, 3.3, 11.0, 15.7, and $20.3 \mathrm{~ns}$ for cells 1 through 6 , respectively. The ideal timing used on RITS-6 is a 4.4-ns delay between successive cells.) Figure 8 is a particle snapshot taken just before cell 4 fires and shows electrons penetrating that cavity to $r \sim 40 \mathrm{~cm}$. This is much deeper penetration than electrons achieve under nominal operating conditions. The transverse current and $E$ field inside cavity 4 are shown as functions of time in Fig. 9. There is a period in the pulse 
during which the electron current loses energy to the field $\left(\int \mathbf{J} \cdot \mathbf{E} d^{3} x<0\right)$.

Two additional diagnostics are used to verify that energy transfer is taking place between particles and the electric field in cavities with electron penetration. The first is a combined particle and field diagnostic which calculates the sum of $\mathbf{F} \cdot \mathbf{v}_{p}$ over every particle in a volume, where $\mathbf{F}$ is the electric force and $\mathbf{v}_{p}$ is a particle velocity. Net energy is transferred from particles to the field if $\mathbf{F} \cdot \mathbf{v}<0$. The second diagnostic computes the Hamiltonian, or the sum

of the kinetic and potential energies $[H=e \Phi+m(\gamma-$ 1)], for individual particles so that energy changes as a result of field interactions can be viewed. The Hamiltonian diagnostic was computed in particle snapshots at times coincident with the sharp increase in the electric field observed in Fig. 9. The particles farthest from the axis in cell 4 (to $y=-42 \mathrm{~cm}$ ) rapidly lose approximately $10 \%$ of their total energy. (Energy loss occurs between 41.25 and 41.5 ns, relative to the time axis in Fig. 9.) Results for the $\mathbf{F} \cdot \mathbf{v}_{p}$ diagnostic are shown in Fig. 10. Here, the values of
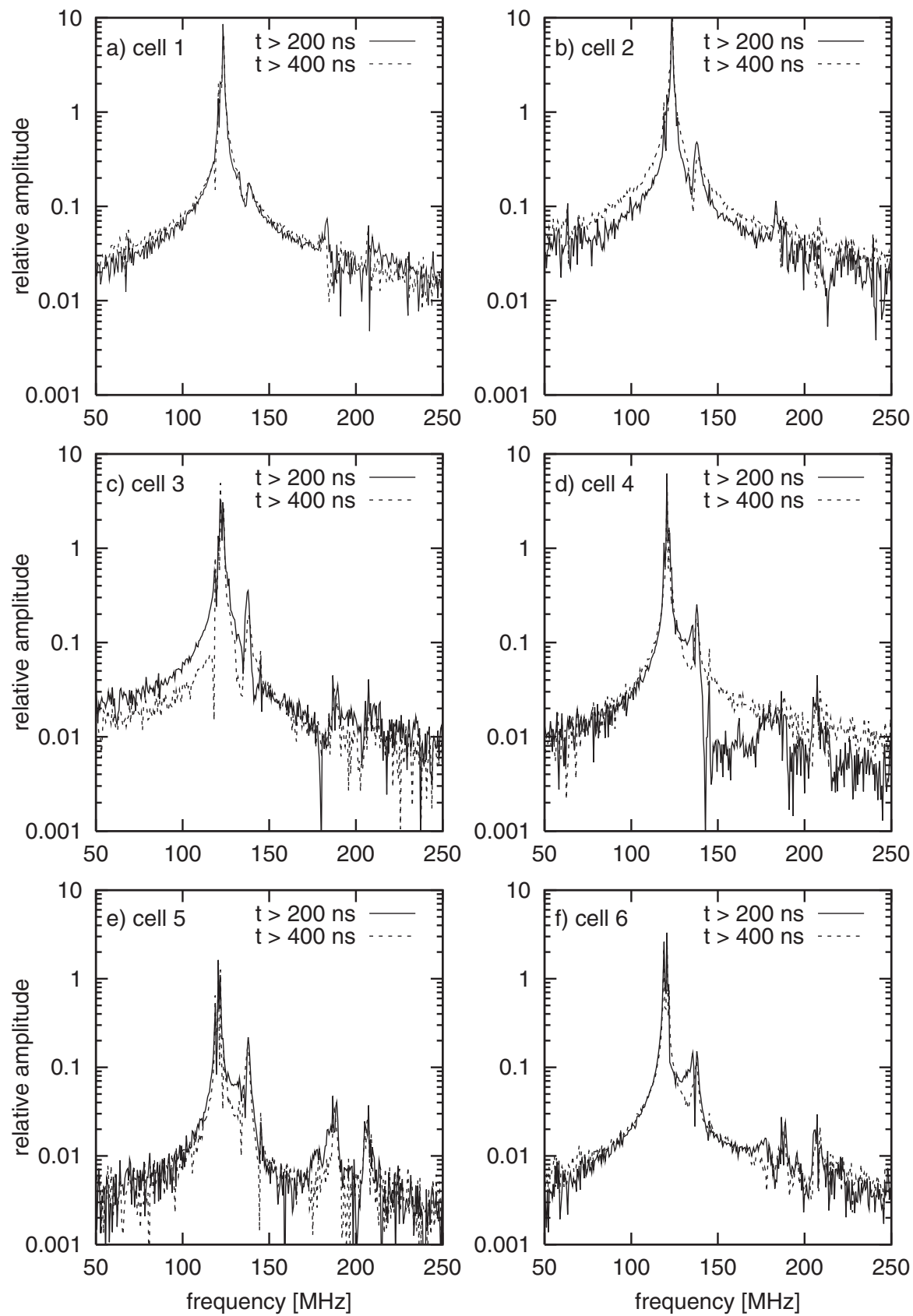

FIG. 7. Electric field spectra generated using a dipole current source located in cavity 2 with a frequency ramp from 116 to $141 \mathrm{MHz}$ over $100 \mathrm{~ns}$. Spectra are calculated for each RITS-6 cell for two time windows, $200 \mathrm{~ns}-2 \mu \mathrm{s}$ and $400 \mathrm{~ns}-2 \mu \mathrm{s}$, to view the decay of the resonances. 
$\mathbf{F} \cdot \mathbf{v}_{p}$ are plotted over time for cavities 3 and 4 (in the volume $-1 \leq x \leq 7$ and $-50 \leq y \leq-20$ in each cavity, chosen to coincide with the particle illustration in Fig. 8).

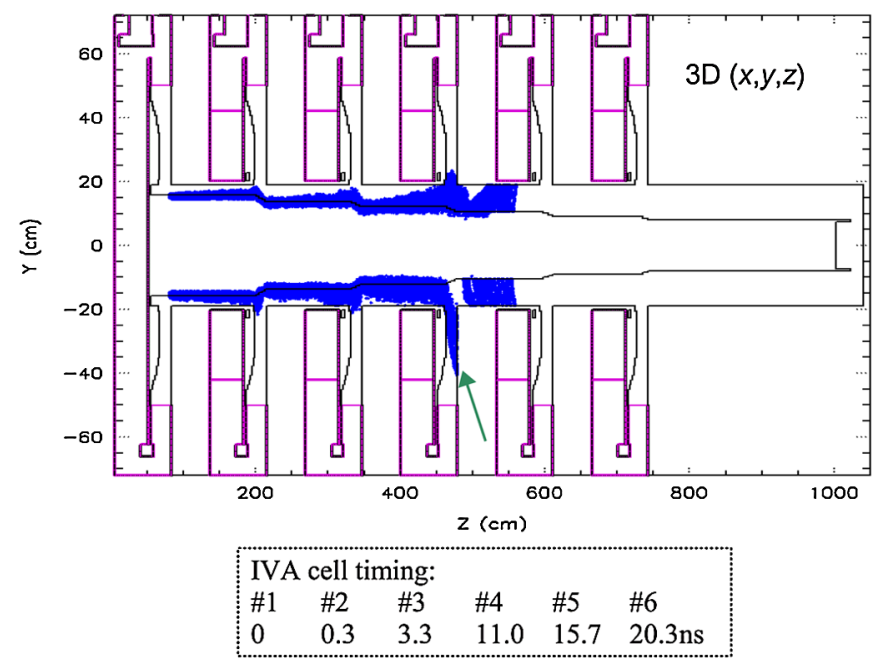

FIG. 8. (Color) Particle snapshot for a simulation with a $7.7 \mathrm{~ns}$ delay between the firings of cells 3 and 4 taken just before IVA cell 4 fires. A green arrow indicates electron macroparticles at very large radius in the cavity of cell 4 . Electron flow asymmetries are due to pulse asymmetries in the MITL.

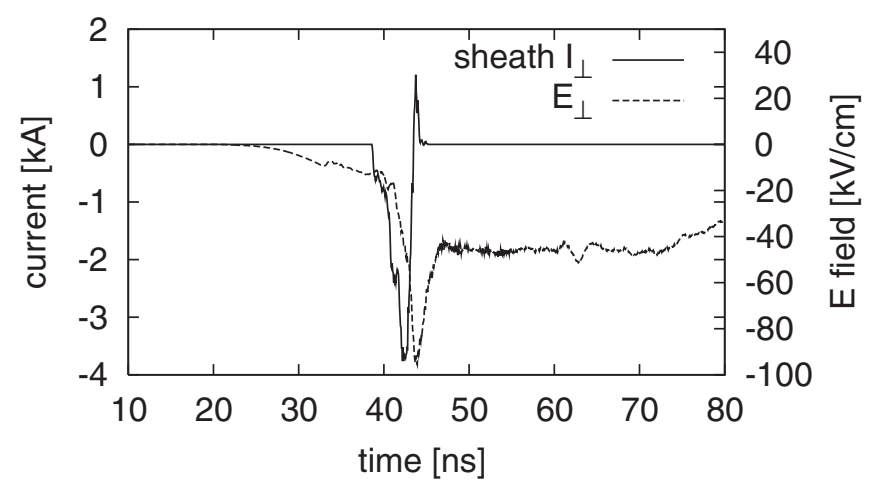

FIG. 9. Transverse current and field in IVA cell 4 for a 7.7 ns between the firings of cells 3 and 4 . The values are measured at $x=25 \mathrm{~cm}$ and $y=0$ in Fig. 8 .
While no net energy transfer is recorded inside cavity 3 , a negative change in $\mathbf{F} \cdot \mathbf{v}_{p}$ is recorded for cavity 4 consistent with the timing of the transient current and field pulses in Fig. 9. The voltages across the cavity are also plotted over time for cavities 3 and 4 in Fig. 10. In nominal cell operation, these voltages are affected by the line impedances and the other cell waveforms but are not heavily modulated. The waveform in Fig. 10(a) for cavity 3 is typical for RITS-6. The waveform in Fig. 10(b) for cavity 4 shows the arrival of the upstream pulses in the cavity prior to firing and the large oscillations that are a result of the cavity excitation.

These oscillations are also seen in $B$-field probes located in each cavity, which are compared to a simulation using nominally spaced pulses in Fig. 11 . The $B$-field probes provide a more direct comparison to the data in Fig. 2(a). The results from the delayed cell-firings show a resonance near $120 \mathrm{MHz}$ initiating in cavity 4 and propagating downstream. Because of the progressive amplification, the mode amplitudes in cavities 5 and 6 are larger than in cavity 4 . The 120-MHz oscillation is consistent with Figs. 2(a) and 7 and with related studies of resonance amplification by means of energy exchange with the electron sheath in a MITL, where it was found that the lowest order mode has the highest growth rate [14].

The dose rate for self-pinch diodes had been measured to scale roughly as $I V^{2.2}$ [15]. This relation is applied to the simulations of the delayed cell firings and the nominal timings to provide a rough estimate of the dose rates for both cases. The results are plotted in Fig. 12. The cavity oscillations due to the delayed cell timings cause dose-rate oscillations similar to those observed in Fig. 2(b). Note that no effort was made to exactly match the data in the specific shot shown in Fig. 2.

Changes in any accelerator parameter which reduce the number of high-momentum electrons in the vicinity of a cell before it fires lower the probability of exciting cavity oscillations. For example, a delay of $5 \mathrm{~ns}$ between the firings of cells 3 and 4 did not result in oscillations. An increase in the pulse rise from 12 to $30 \mathrm{~ns}$, while keeping the delayed timings, also did not result in oscillations. As
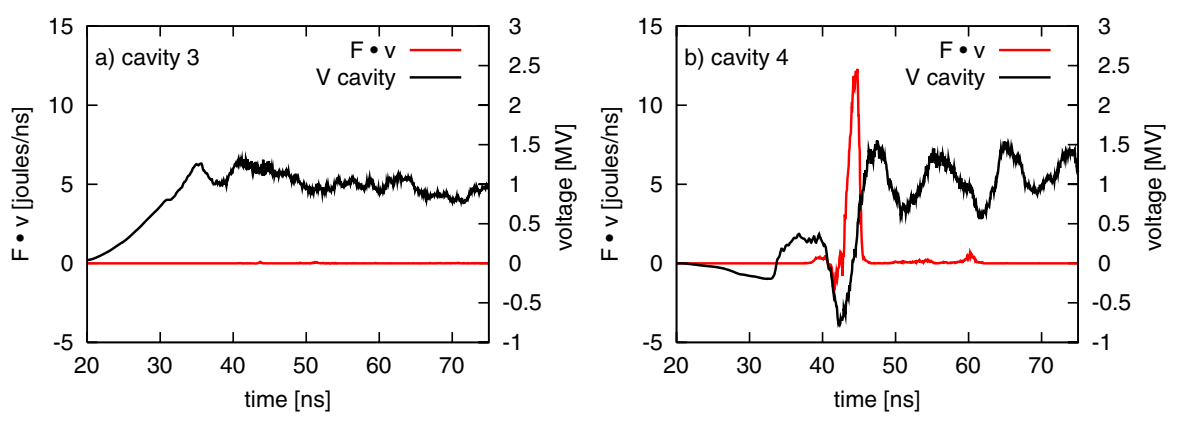

FIG. 10. (Color) Energy exchange $\left(\mathbf{F} \cdot \mathrm{v}_{p}\right)$ between electrons and the electric field recorded in cavities 3 (a) and 4 (b) for a simulation with a $7.7 \mathrm{~ns}$ delay between the firings of those cells. The cell voltages recorded inside the cell cavity $(x=30 \mathrm{~cm}, y=0)$ are also shown. 

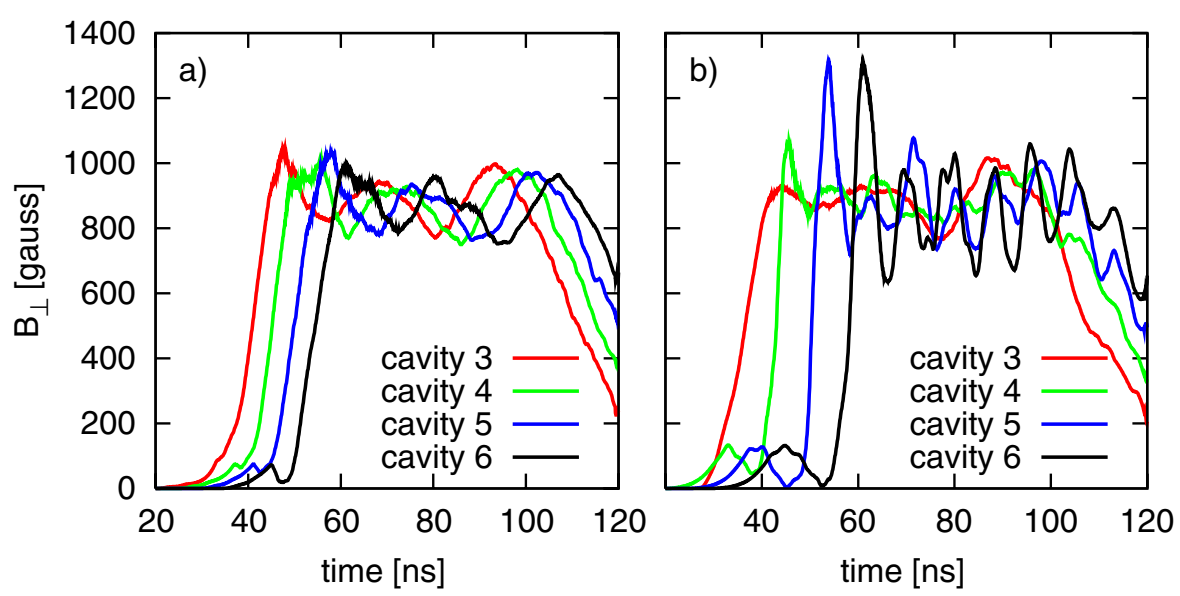

FIG. 11. (Color) Simulated $B$-field probes in IVA cells 3-6 for (a) cell firings spaced 3.3 ns apart (nominal timing) and (b) cell firings spaced 7.7, 4.7, and 4.6 ns apart for cells 3-6, respectively. Probes are located 180 degrees from the radial feed.

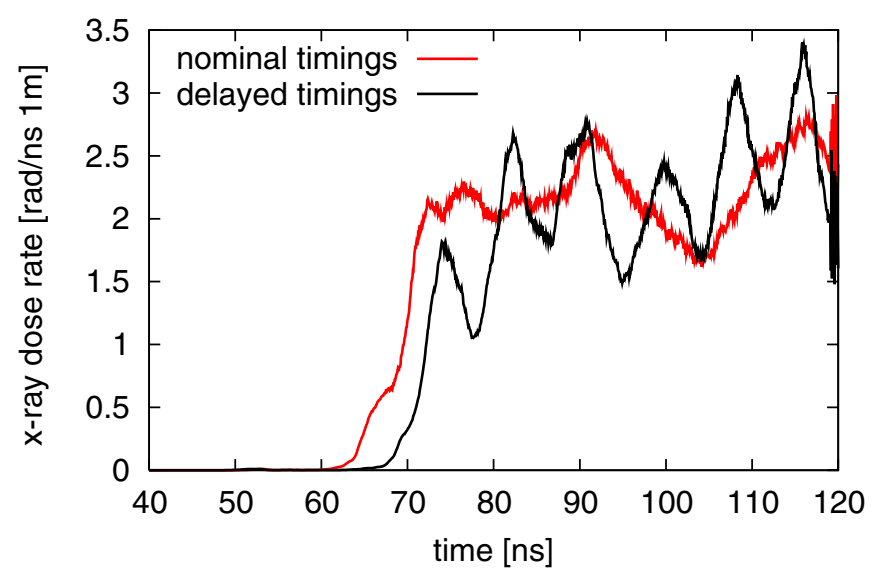

FIG. 12. Simulated x-ray dose rate for uniform cell firings spaced $3.3 \mathrm{~ns}$ apart (nominal timing) and cell firing times of 0 , $0.3,3.3,11.0,15.7$, and $20.3 \mathrm{~ns}$ (delayed timings).

the pulse rise approaches the nominal delay timing between cavities, the more accurate these timings must be to avoid oscillations. The relatively long pulse rises of the other IVA accelerators, e.g., Hermes-III [16], Sabre [17], and Mercury, may be the reason they have not observed this phenomenon.

\section{CONCLUSIONS}

It has been shown that the IVA architecture used in the design of Sandia National Laboratories' RITS-6 accelerator, and other recent designs of pulsed-power radiographic systems, is susceptible to oscillations in the voltage pulse which potentially degrade performance. The frequency and method of excitation for these oscillations has been studied here with the aid of particle-in-cell simulations.

Treating the IVA cells as resonant cavities, modes were calculated for the specific IVA design of RITS-6. The highest-quality mode calculated agrees with the measured frequency $(\approx 120 \mathrm{MH} z$ ) in current monitors in the IVA cavities and along the transmission line.

Particle-in-cell simulations demonstrated how the resonance could be excited. 3D simulations of RITS-6 showed that during the pulse rise, as magnetic insulation is being established, some electrons traverse the anode-cathode gap in the vicinity of the voltage adder cells. When conditions permit, high-momentum electrons may penetrate the cavities of the adder cells to large radius where they can transfer energy to the fields and excite rf resonances. The condition in the RITS-6 simulation is a delayed firing of one of the adder cells. The mode then experiences progressive amplification when it propagates past successive IVA cells.

It was also seen that oscillations were not excited when using the nominal cell timings of RITS-6. Nor were they excited when the pulse rise was significantly increased. This may explain the absence of voltage oscillations in previous IVA-based accelerators which had pulse rises greater than 20 ns. The sharp 12-ns pulse rise of RITS-6, for which the pulse rise approaches the cell timings, makes it more susceptible to this type of oscillation. More precise control of the delay between cell firings will mitigate IVAcavity oscillations in RITS-6 and future short-pulse IVAbased accelerators.

\section{ACKNOWLEDGMENTS}

This work is supported by Sandia National Laboratories and the U.S. Department of Energy and the U.K. Ministry of Defense, under Contract No. DOA-8910 and AWE under PALD 760. Sandia is a multiprogram laboratory operated by Sandia Corporation, a Lockheed Martin Company, for the United States Department of Energy's National Nuclear Security Administration under Contract No. DE-AC0494AL85000. 
[1] I. Smith, Phys. Rev. ST Accel. Beams 7, 064801 (2004).

[2] I. Smith, V. Bailey, J. Fockler, J. Gustwiller, D. Johnson, J. Maenchen, and D. Droemer, IEEE Trans. Plasma Sci. 28, 1653 (2000).

[3] I. Smith et al., in Proceedings of the 14th IEEE International Pulsed Power Conference, 2003 (IEEE, New York, 2003), Vol. 1, pp. 371-378.

[4] R. Commisso et al., in Proceedings of the 14th IEEE International Pulsed Power Conference, 2003 (Ref. [3]), Vol. 1, pp. 383-386.

[5] D. Johnson et al., in Proceedings of the 15th IEEE International Pulsed Power Conference (IEEE, New York, 2005), pp. 314-317.

[6] J. Creedon, J. Appl. Phys. 48, 1070 (1977).

[7] C. Mendel, D. Seidel, and S. Rosenthal, Laser Part. Beams 1, 311 (1983).

[8] R. Fowler and L. Nordheim, Proc. R. Soc. A 119, 173 (1928).

[9] J. Creedon, J. Appl. Phys. 46, 2946 (1975).
[10] C. Mendel, J. Appl. Phys. 50, 3830 (1979).

[11] N. Bruner, C. Mostrom, D. Rose, D. Welch, V. Bailey, D. Johnson, and B. Oliver, in Proceedings of the 16th IEEE International Pulsed Power and Plasma Science Conference (IEEE, New York, 2007), pp. 807-810.

[12] T. Hughes, S. Yu, and R. Clark, Phys. Rev. ST Accel. Beams 2, 110401 (1999).

[13] B. Goplen, L. Ludeking, D. Smithe, and G. Warren, Comput. Phys. Commun. 87, 54 (1995).

[14] R. Lemke, J. Appl. Phys. 66, 1089 (1989).

[15] D. Hinshelwood et al., IEEE Trans. Plasma Sci. 35, 565 (2007).

[16] J. Ramirez et al., in Proceedings of the 7th IEEE International Pulsed Power Conference (IEEE, New York, 1989, Vol. 1, p. 26.

[17] M. Cuneo, J. Poukey, C. Mendel, S. Rosenthal, D. Hanson, J. Smith, J. Maenchen, D. Wenger, and M. Bernard, in Proceedings of the 9th IEEE International Pulsed Power Conference (IEEE, New York, 1993), Vol. 1, p. 423. 